http://jmscr.igmpublication.org/home/ ISSN (e)-2347-176x ISSN (p) 2455-0450 crossref DOI: https://dx.doi.org/10.18535/jmscr/v7i9.18

\author{
Journal Of Medical Science And Clinical Research

\title{
Maternal Outcome in Pregnancies with Thrombocytopenia
}

\author{
Authors \\ Dr Rinku G ${ }^{\mathbf{1}}$, Dr. M I Geetha ${ }^{2 *}$ \\ ${ }^{1}$ Assistant Professor, Dept. of O \& G, SATH, Govt. Medical College, Trivandrum. \\ ${ }^{2}$ Associate Professor, Dept. of O \& G, SATH, Govt. Medical College, Trivandrum. \\ *Corresponding Author \\ Dr M I Geetha \\ Associate Professor, Dept. of O \& G, SATH, Govt. Medical College, Trivandrum, India
}

\begin{abstract}
Background: Thrombocytopenia is a common occurrence in pregnancy. Although pregnancy is associated with physiological changes in platelet count, several pathological conditions cause thrombocytopenia, which can have a significant impact on the mother and the baby. The present study aims at the maternal outcome of pregnancies in patients with thrombocytopenia during pregnancy.

Materials and Methods: Comparative cross sectional survey conducted in SAT Hospital for a period of one year.

Results: In our study gestational thrombocytopenia constitutes $40 \%$ of cases and majority are mild type. Moderate and severe types were constituted by Pre eclampsia 10.8\%, HELLP syndrome 17.4\%, ITP $11.4 \%$ SLE 9\% and DIC especially following grade III abruption. Out of moderate to severe cases $13.8 \%$ developed post-partum hemorrhage requiring blood and blood products transfusion. Maternal mortality (1 case) occurred in SLE group due to intractable pulmonary edema. Cesarean section rate was more in thrombocytopenia group.

Conclusion: Gestational Thrombocytopenia group had a favorable outcome in our study. The other group constituted by pre eclampsia SLE, ITP, DIC had complications like PPH, abruptions, DIC requiring multidisciplinary approach.

Keywords: Thrombocytopenia, ITP (immune thrombocytopenic purpura), SLE (systemic lupus erythamatosis).
\end{abstract}

\section{Introduction}

A mild thrombocytopenia is relatively frequent during pregnancy and has no consequences for either the mother or the fetus. But it may result from a range of pathologic conditions requiring closer monitoring and therapy. It is defined as a platelet count $<1.5 \mathrm{~L}$ per $\mathrm{mm}^{3}$ and is second only to anemia.

Incidental thrombocytopenia of pregnancy gestational thrombocytopenia- accounts for 70-
$80 \%$ of cases and it occurs in the mid -second, third trimester of pregnancy ${ }^{1}$. It may result from various mechanisms including hemodilution, decreased platelet production and accelerated clearance $^{6}$ and diagnosis is only of exclusion.

Counts from $1 \mathrm{~L}$ to $1.5 \mathrm{~L}$ per $\mathrm{mm}^{3}$ are mildly depressed, 50000 to $1 \mathrm{~L}$ per $\mathrm{mm}^{3}$ moderately depressed and $>50000$ are severely deppressed ${ }^{3}$. The most common cause is gestational thrombocytopenia. Hypertensive disorders 
account for $21 \%$. Thrombocytopenia occurs more commonly in patients with eclampsia (30\%) than in patients with both mild and severe forms of preeclampsia $(15-18 \%)^{4}$ of the patients who have severe pre-eclampsia $4-12 \%$ will manifest criteria of HELLP Syndrome ${ }^{5}$.

Immune mediated thrombocytopenia including idiopathic thrombocytopenic purpuras is responsible for $4.1 \%$ of cases. Other less common causes include rheumatological diseases (SLE), DIC, TTP, APLA, HIV infection and medications. ITP is the most common cause of thrombocytopenia at less than 20 weeks gestation and aim of treatment is to increase platelet count and to ensure safe delivery. Maternal anti platelet antibodies can cross the placenta and studies indicate that $12-15 \%$ of infants born to these mothers develop severe thrombocytopenia.

TTP is a rare but life threatening disease that should be suspected with thrombocytopenia and microangiopathichemolytic anemia and treatment with IVIG should begin immediately to prevent maternal death ${ }^{8}$. Occasionally a previously undiagnosed congenital platelet disorder maybe recognized for the first time during pregnancy. At delivery placental separation occurs at a time when normal blood flow is approximately $700 \mathrm{ml}$ per minute. This flow is dampened by uterine contraction leading to myometriyal compression and occlusion by physiologic thrombosis of the open maternal vessels. Defect in either mechanism to arrest uterine bleeding leads to potentially lethal haemorrhage. Therefore when thrombocytopenia is diagnosed in pregnancy the women should undergo further clinical and laboratory assessment to determine the cause.

\section{Objective}

\section{Primary Objective}

To study the maternal outcome in patients with hrombocytopenia and comparing with those without thromboicytopenia.

\section{Secondary Objective}

To compare the maternal outcome of different etiologies of thrombocytopenia.

\section{Materials and Method}

Study Design- Comparative cross sectional survey

Study population include all pregnant women with thrombocytopenia delivering in SAT Hospital, Govt. Medical College, Trivandrum for one year and comparing with equal number of pregnant patients without thrombocytopenia and hypertensive disorders who deliver during the same period.

Sample size is calculated by the formula:

$$
\begin{aligned}
& \mathrm{N}=2 \mathrm{pq}(\mathrm{Z} \alpha+Z \beta)^{2} \\
& \left(\mathrm{P}_{1}-\mathrm{P}_{0}\right) \\
& \mathrm{P}=\mathrm{Po} / 2, \mathrm{q}=1-\mathrm{p} \\
& \mathrm{P}_{0}=\text { Control group response } \\
& \mathrm{P}_{1}=\text { Study group response }=\quad \underline{\mathrm{P}_{0}} \underline{\underline{\mathrm{R}}} \\
& 1+\mathrm{P}_{0}(\mathrm{RR}-1)
\end{aligned}
$$

200 cases of thrombocytopenia patients and 200 consecutive pregnancies without thrombocytopenia and hypertension

The clinical details of all women were collected by reviewing their hospital records, labour records, and the cases will be analyzed according to the history and the medical records. Following clinical characteristics - maternal age, previous gestations, parity, gestational age, birth weight, cause of thrombocytopenia were evaluated. Obstetrics risk factors- GDM, overtdiabetis, previous CS, maternal anemia, hydramnious, ologamnious, multiple pregnancies, thyroid disease were examined including the following.

$>$ Requirement of Blood and platelet transfusion

$>$ Placental abruption, Intra uterine death

$>$ Labour induction, Mode of delivery

$>\mathrm{PPH}$, episiotomy haematoma, anaesthetic complications

Statistical analysis done by Chi square test, odds ratio and logistical regression. 


\section{Ethical issues}

Being comparative cross sectional survey, permission from hospital authorities is required for collecting information.

\section{Results}

\section{Observation and Results}

\section{Age of the patients}

Maximum patients $(85 \%)$ in the thrombocytopenia group belong to the age group 20-29 years. The controls too had maximum number in the same age group $(89.8 \%)$ but in the age group of 30-34 years, there were 20 patients in the case against 6 in the control.

\begin{tabular}{|l|c|c|c|c|}
\hline \multirow{2}{*}{$\begin{array}{l}\text { Age } \\
\text { group }\end{array}$} & \multicolumn{2}{|c|}{ Case } & \multicolumn{2}{c|}{ Control } \\
\cline { 2 - 5 } & Number & Percentage & Number & Percentage \\
\hline$<19$ & 0 & 0 & 7 & 4.2 \\
\hline $20-24$ & 75 & 44.9 & 86 & 51.5 \\
\hline $25-29$ & 67 & 40.1 & 64 & 38.3 \\
\hline $30-34$ & 20 & 12 & 6 & 3.6 \\
\hline$>35$ & 5 & 3 & 4 & 2.4 \\
\hline Total & 167 & 100 & 167 & 100 \\
\hline
\end{tabular}

\section{Gestational age of diagnosis of} thrombocytopenia

$76 \%$ of the cases of thrombocytopenia were diagnosed in the third trimester. ITP and SLE cases had thrombocytopenia presenting in the first and second trimester. Abruption which was an important caused of acute onset of thrombocytopenia also occurred more towards the third trimester.

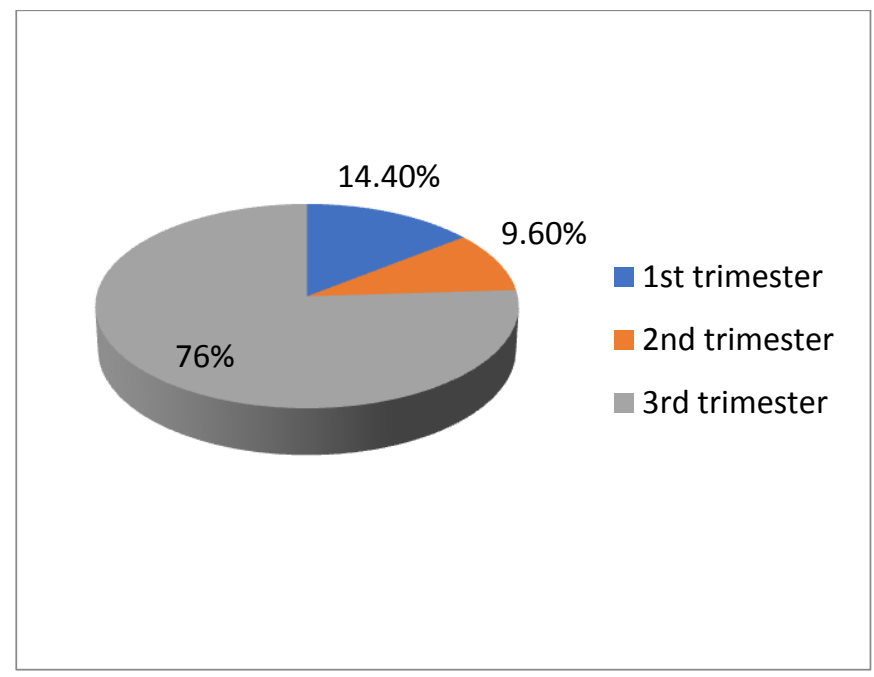

Gestational age of thrombocytopenia

\section{Booked cases vsun booked cases}

One patient among the control group was unbooked and all cases among the thrombocytopenia group were booked cases.

\section{Referred cases}

$59.9 \%$ of patients among the cases were referred to our hospital while only $18.6 \%$ of the control group were referred.

\section{Parity}

No significant difference noted in the case and control group $(p$ value $=0.219)$. however, preeclampsia is more common in Primigravida.

\begin{tabular}{|l|c|c|c|c|c|c|}
\hline \multirow{2}{*}{ Referred } & \multicolumn{2}{|c|}{ Case } & \multicolumn{2}{c|}{ Control } & \multicolumn{2}{c|}{ Total } \\
\cline { 2 - 7 } & $\mathrm{N}$ & $\%$ & $\mathrm{~N}$ & $\%$ & $\mathrm{~N}$ & $\%$ \\
\hline Primi & 95 & 56.9 & 106 & 63.5 & 201 & 60.2 \\
\hline Multi & 72 & 43.1 & 61 & 36.5 & 133 & 39.8 \\
\hline Total & 167 & 100 & 167 & 100 & 334 & 100 \\
\hline
\end{tabular}

\section{Gestational age at delivery}

There was no significant difference among the two groups in terms of gestational age at delivery, majority of patients in both the arms delivered at 37-40 weeks.

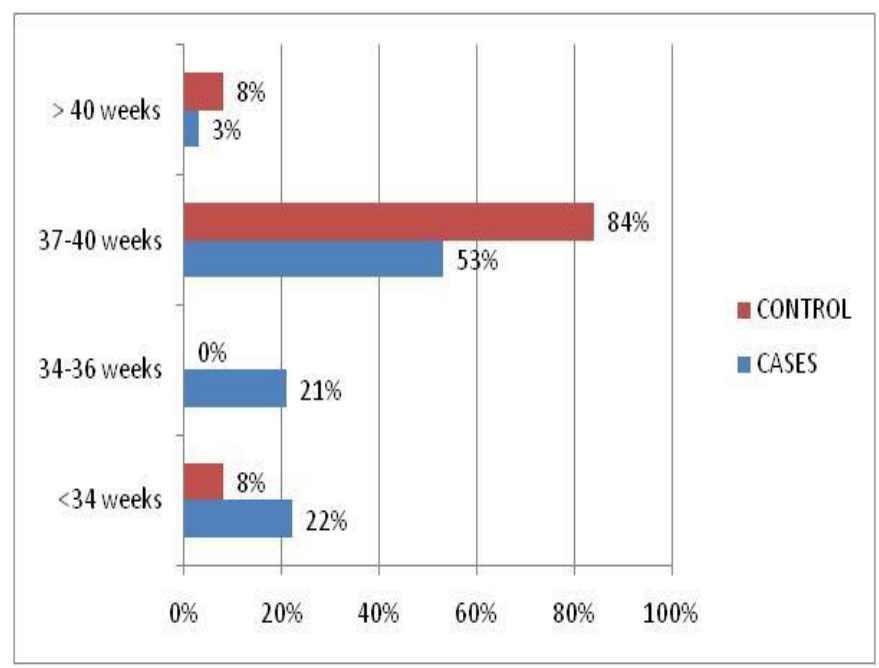

\section{Severity of thrombocytopenia}

Majority were mild cases of thrombocytopenia $(55 \%)$. The moderate $(33 \%)$ and $(12 \%)$ severe forms were constituted by ITP, SLE and DIC especially following cases of grade 3 abruption. 


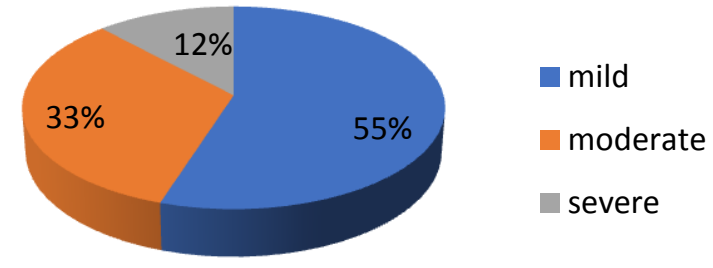

VIII. Causes of thrombocytopenia

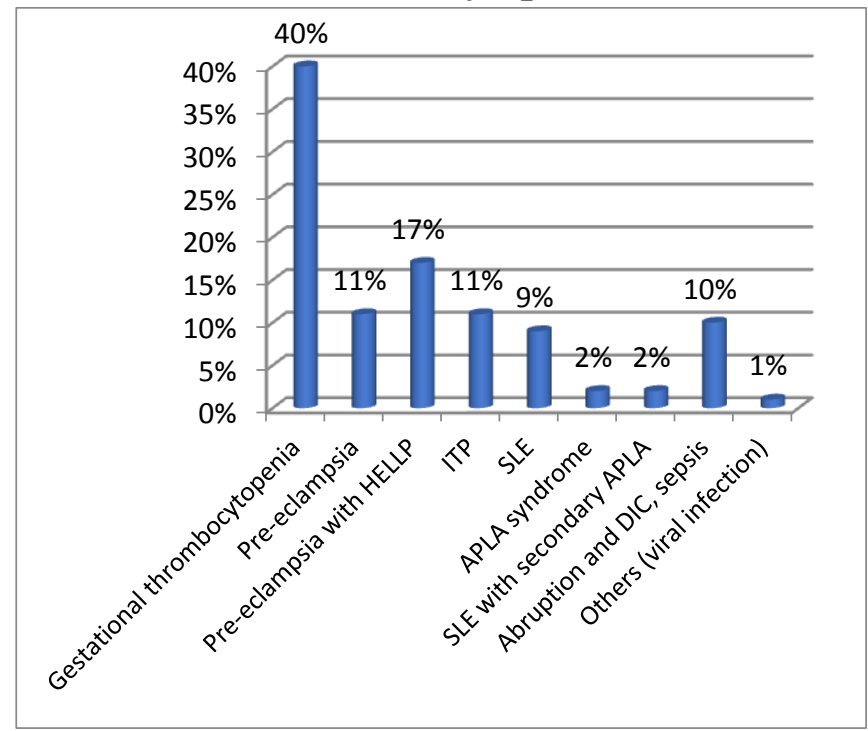

There was overlap between cases of SLE, ITP and pre-eclampsia as some of these patients had all these disorders together. Abruption was the most important cause of DIC and associated thrombocytopenia. Some patients with preeclampsia also showed evidence of DIC.

\section{Gestational hypertension and its association with thrombocytopenia}

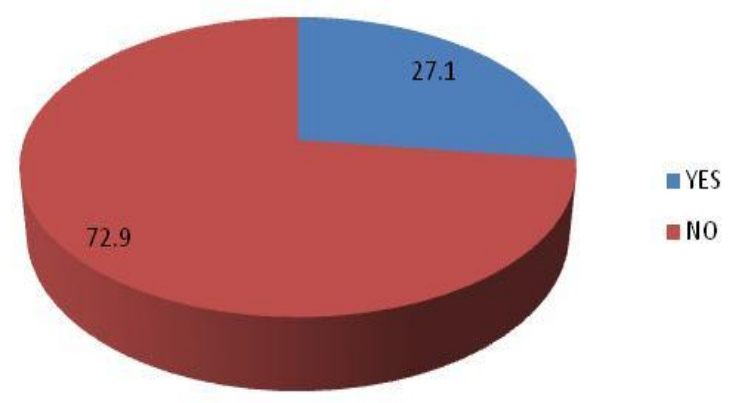

$27.1 \%$ of patients in the case group had gestational hypertension.
X. Thrombocytopenia and maternal risk factors

No significant difference was noted between the cases and the control group with respect to maternal risk factors like diabetes mellitus, maternal hypothyroidism, anaemia, oligohydramnios, polyhydramnios, multiple pregnancy and preterm labour.

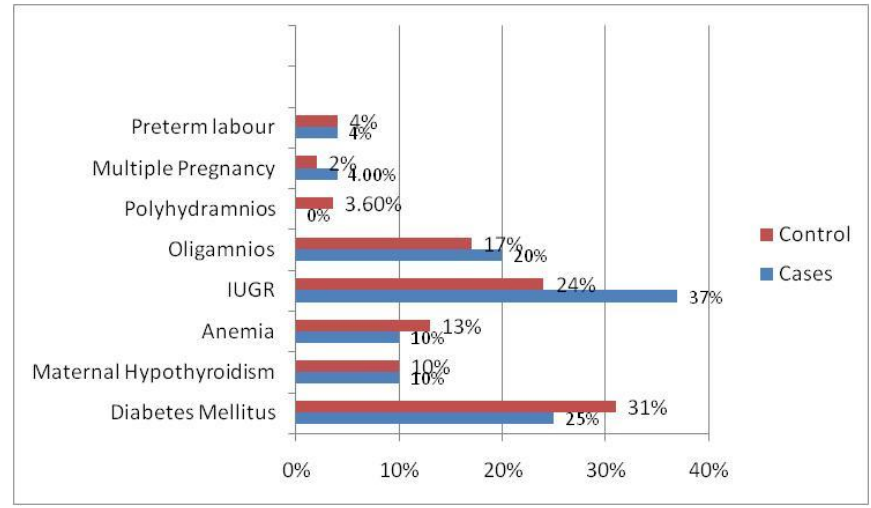

\section{Abruption as a cause of thrombocytopenia}

There were 20 cases of grade III abruption, majority of which required blood transfusion and ICU management for correction of hypotension, coagulation failure and renal compromise.

\section{Mode of delivery}

There were a greater number of caesarean sections in the thrombocytopenia group $54.5 \%$ vs $25.1 \%$ in the control group.

\section{PPH and types of PPH}

There were 23 cases $(13.8 \%)$ of $\mathrm{PPH}$ in the thrombocytopenia group vs 4 cases $(2.4 \%)$ in the control group. The various factors contributing to PPH were pre-eclampsia, HELLP, DIC and coagulation failure especially, following abruption which resulted in both atonic PPH and PPH due to DIC.

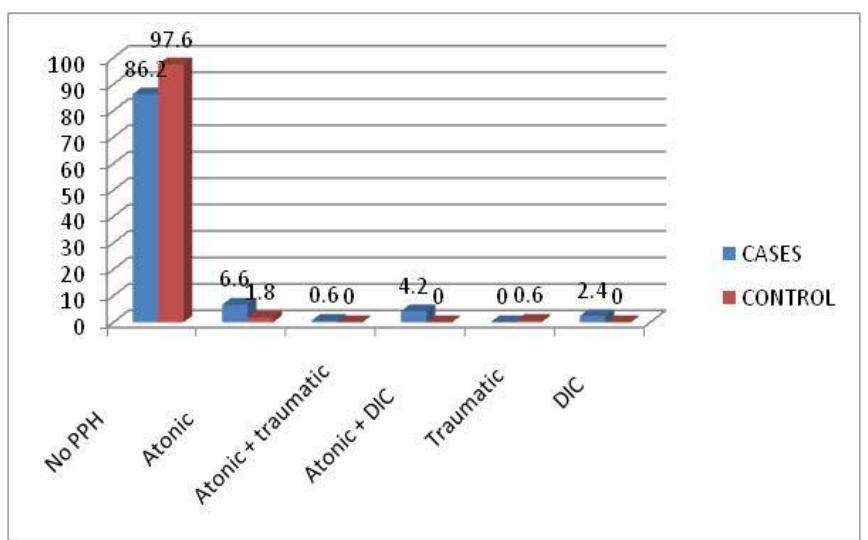


Other complications noted in the case group was seizures 6 cases $(3.2 \%)$, renal complications and oliguria 5 cases $(2.8 \%)$, bleeding and coagulation abnormalities 15 cases (9\%), sepsis cases 1 $(0.57 \%)$, pulmonary oedema 1 cases $(0.57 \%)$ and 1cases $(0.57 \%)$ case of maternal death while no such maternal complications were observed in the control group.

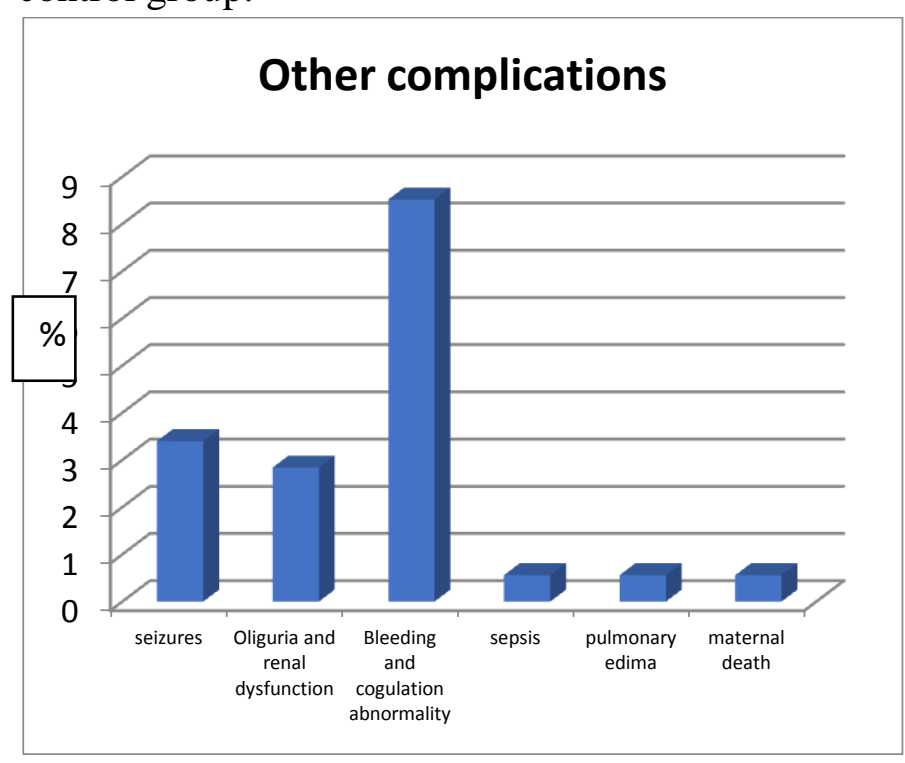

The case of maternal mortality was an SLE patient who went into DKA and thrombocytopenia and died due to intractable pulmonary oedema during LSCS done for failed induction. She had received 3 doses of methyl prednisolone prior to her LSCS to improve her platelet count.

There were 38 cases of abruption in the case group (Grade I-3 cases, Grade II-15 cases, Grade III-20 cases) compared to only 2 cases (Grade II) in the control group.

\section{Discussions}

Our study aims at the maternal outcome of pregnancies complicated by thrombocytopenia. All the causes of thrombocytopenia in pregnant ladies admitted to our labour room were studied including acute onset of thrombocytopenia in patients presenting with acute causes like abruption and DIC.

$55.1 \%$ of cases had only mild thrombocytopenia and $76 \%$ of the cases of thrombocytopenia were diagnosed or had their first presentation in the third trimester ${ }^{13}$. The severe form of thrombocytopenia (count $<50000 / \mathrm{cmm}$ ) were mostly constituted by ITP patients (11\%) and SLE (9\%) patients, pre eclampsia with HELLP (17\%) (similar to the study by Vyas. Retal - GT $44.6 \%$, HELLP 22\%). Also causes of abruptio placentae with DIC had a severe thrombocytopenia with associated coagulation abnormalities. There were 2 cases of viral infections (dengue fever) with thrombocytopenia managed with supportive measures. Hence the importance of routine examination of platelet counts in all the trimesters to diagnose the condition and reduce maternal and fetal complications.

In our study, gestational thrombocytopenia is the most common cause of thrombocytopenia (40\%). Around $10.8 \%$ cases were due to pre-eclampsia and $17.4 \%$ constituted by HELLP syndrome. Many patients had co-existing multiple conditions like SLE, ITP and pre-eclampsia.

Autoimmune causes like ITP (11.4\%), SLE(9\%) and SLE with 2 degree $\operatorname{ALPA}(2.4 \%)$ and 1 degree $\operatorname{ALPA}(2.4 \%)$ also constituted a sizeable portion. Being a tertiary case centre with an efficient haemetology department in our medical college, we had many such patients referred to us, which resulted in a multidisciplinary and efficient management system for these patients.

Regarding gestational age at delivery, $44 \%$ of the cases delivered before 36 weeks of gestation compared to only $8.4 \%$ among controls. This was because the pre-eclampsia, HELLP, APLA $^{9}$ syndrome and abruptio placentae cases had to be terminated before term for both maternal and fetal sake. These patients had placental insufficiency and FGR which also demands earlier termination of pregnancies.

There was more caesarean section in the above case group, $54.5 \%$ vs $25.1 \%$ in the control group. There were a greater number of previous CS cases among the thrombocytopenia patients.

Postpartum haemorrhage $(13.8 \%)^{12}$ among case group vs $(2.4 \%)$ among controls was significant. Factors contributing were pre-eclampsia, HELLP, DIC, coagulation failure, especially following 
abruption which resulted in both above PPH and PPH due to DIC.

There were other complications noted in the case group- seizures (6 cases), oliguria and renal dysfunction- 5 cases, bleeding and coagulation abnormality- 15 cases, sepsis-1, pulmonary oedema-1 and one case of maternal death due to intractable pulmonary oedema in an SLE patient. No such maternal complications were observed in the control group.

Blood and blood products transfusion like FFP, cryoprecipitate, platelet rich plasma, platelet concentrate were given in Abruption Grade III (12\%), Grade II (9\%), patients with DIC, HELLP syndrome (17.4\%) and also in ITP patients intractable to medical management.

\section{Conclusion}

In general, the gestational thrombocytopenia (GT) and ITP groups had a favorable outcome in our study. Gestational thrombocytopenia is not associated with an increased incidence of pregnancy related complications or with the delivery of a thrombocytopenic offspring. ITP and SLE tend to occur in younger women. The rarer and more serious group of causes of thrombocytopenia including DIC, familial TTP, APLA syndrome etc are associated with placental abruption, low Apgar scores and stillbirths.

Higher rates of preterm deliveries $(<34$ weeks $22.15 \%, 34-37$ weeks $21.55 \%$ ) were observed among patients with moderate to severe thrombocytopenia. This is due to labour induction, abruption, IUGR and for maternal sake in severe forms of pre-eclampsia and HELLP syndrome. The management of SLE, ITP patients etc were done in collaboration with the Haematology Department Multidisciplinary approach of management was undertaken whenever needed. Adverse perinatal outcome was mostly associated with abruption, DIC, APLA syndrome and HELLP syndrome.

Careful surveillance is required for all such highrisk pregnancies for early detection and treatment of possible complications, in order to reduce to maternal and neonatal morbidities.

\section{Recommendation}

Since majority of cases of thrombocytopenia are diagnosed during pregnancy, it should be made a routine to check the platelet count in the first trimester of pregnancy itself. The etiology of thrombocytopenia is to be determined and treatment to be initiated in collaboration with other departments like Haematology, Transfusion medicine, Nephrology, Medical Gastroenterology etc.

\section{Reference}

1. Sainio S, Kekomaki R, Riikonen S, Teramo K. Maternal thrombocytopenia at term- a pmpulation based study. Actaobstgynecolscand 2000; 79(9);744749.

2. Magann IT et al. Twelve steps to optimal management of HELLP syndrome. Clin Obstet Gyncol 1999; 42:532-50.

3. Provan D et al (2010) international consensus report on the investigation and management of primary immune thrombocytopenia. Blood I 15:168-186.

4. Adams 1)0T et al. Maternal thrombocytopenia in pregnancy. Cliniscs in laboratory medicine, 2013-06-01, Volume 25. Issue 2, Pages 327-341.

5. Sullivan CA et al. Management of the obstetric patient with thrombocytopenia. ClinObstetGynecol 1995;38:521-34.

6. Rodger $\mathrm{M}$ et al. Hematological problems in obstetrics. Best practice \& Research: Clinical Obstetrics \&Gynaecology, 20d1507-01, Volume 2.9. Issue 5. Pages 671684.

7. Ying-Hsuan Lin et al. Perinatal outcome in normal pregnant women with incidental thrombocytopenia at delivery. Taiwanese Journal of Obstetrics and Gynaecology, 2013-19'01, Volume 52, Issue 3, Pages 341-350. 
8. Meyer o, (2003) Lupus et autresconnectivities et vie hormonale, Gynecologie Obstetrique \& Fertilite.. 31, 746-756. Doi: 10.1016/S 12979589(03)00203-0.

9. Errarhay. $\mathrm{s}$ et al. Antiphospholipid antibody syndrome and pregnancy. Open Journal of Obstetrics and Gynecology Vol. 3 No. 4 (2013), Article ID: 31566, 3 pages DOI :10.4236/ojog.2013.34071.

10. Arvieux, J. and Machulla..E.(2002) Le syndrome des antiphon. sphoiipides. Annales de Cardiologieet d Ange-iologie, 51J 46-151. Doi: 10.1016/S00033928(02)00087-2.

11. Cleary KL et al. (2009) Pre-eclampsia and the kidney. SeminPerinatol 33:I 73-178.

12. Barton JR et al (2004) Diagnosis and management of hemolysis, elevated liver enzymes and low platelets syndrome. Clin Pcrinaiol 31:807 833.

13. Vyas. Retal study of mild versus moderate to severe thrombocytopenia in $3^{\text {rd }}$ trimester of pregnancy in a tertiary care hospital, NHL journal of Medical Sciences/Jan 2014/vol 3/issue.

14. Mamta $\mathrm{S}$ et al. Thrombocytopenia during pregnancy journal of evalution of medical anda dental sciences 2010 month, October Volume; 3 issue:57 page;12956-12960. 